H. Fujimoto

Nagoya Math. J.

Vol. 75 (1979), 71-85

\title{
REMARKS TO THE UNIQUENESS PROBLEM OF MEROMORPHIC MAPS INTO $P^{N}(C)$, III
}

\author{
HIROTAKA FUJIMOTO
}

\section{§1. Introduction}

In the previous papers [3], [4], [5] the author gave some results on the uniqueness of meromorphic maps of $C^{n}$ into the $N$-dimensional complex projective space $P^{N}(C)$ which have the pre-assigned inverse images for some hyperplanes in $P^{N}(C)$. Relating to these results, we attempt in this paper to generalize the following Cartan-Nevanlinna's theorem to the case of meromorphic maps into $P^{N}(C)$.

TheOREm ([2], [6]). If three non-constant meromorphic functions $\varphi, \psi, \chi$ on $C$ have the same inverse images with multiplicities counted for three distinct values, then $\varphi=\psi, \psi=\chi$ or $\chi=\varphi$.

Let us consider $N+2$ hyperplanes $H_{1}, \cdots, H_{N+2}$ in $P^{N}(C)$ located in general position and $N+2$ divisors $\nu_{1}, \cdots, \nu_{N+2}$ whose supports have no common irreducible components. Our purpose is to study the set $\mathscr{F}$ of all meromorphic maps $f$ of $C^{n}$ into $P^{N}(C)$ such that the pull-backs $\nu\left(f, H_{j}\right)$ of the divisors $\left(H_{j}\right)$ on $P^{N}(C)$ by $f$ are equal to $\nu_{j}(1 \leqq j \leqq N+2)$. The main result is stated as follows:

The set $\mathscr{F}$ cannot contain more than $N+1$ algebraically independent maps (c.f., Definition 2.1).

It is possible for $\mathscr{F}$ to contain $N+1$ distinct maps. For example, taking $N^{2}+N$ algebraically independent nowhere zero entire functions $\eta_{j}^{i}(1 \leqq i \leqq N, 1 \leqq j \leqq N+1)$ and putting

$$
k_{j}:=(-1)^{j-1} \operatorname{det}\left(\eta_{\ell}^{k}-1 ; \begin{array}{l}
k \\
\ell=1, \cdots, N \\
\ell=1, \cdots, j-1, j+1, \cdots, N+1
\end{array}\right)
$$

for each $j=1,2, \cdots, N+1$, we define $N+1$ meromorphic maps $f^{i}: C^{n}$

Received March 15, 1978. 
$\rightarrow P^{N}(C)(0 \leqq i \leqq N)$ as

$$
f^{0}=k_{1}: k_{2}: \cdots: k_{N+1}
$$

and

$$
f^{i}=\eta_{1}^{i} k_{1}: \eta_{2}^{i} k_{2}: \cdots: \eta_{N+1}^{i} k_{N+1} \quad(1 \leqq i \leqq N) .
$$

It is easily seen that they satisfy the condition

$$
\nu\left(f^{0}, H_{j}\right)=\nu\left(f^{1}, H_{j}\right)=\cdots=\nu\left(f^{N}, H_{j}\right) \quad(1 \leqq j \leqq N+2)
$$

for hyperplanes $H_{j}=\left\{w_{j}=0\right\}(1 \leqq j \leqq N+1)$ and $H_{N+2}=\left\{w_{1}+\cdots+w_{N+1}\right.$ $=0\}$ in $P^{N}(\boldsymbol{C})$, where $w_{1}: w_{2}: \cdots: w_{N+1}$ is a system of homogeneous coordinates on $P^{N}(C)$.

On the other hand, if the number of given hyperplanes is $N+1, \mathscr{F}$ is very large. In fact, for the above hyperplanes $H_{j}(1 \leqq j \leqq N+1)$ and divisors $\nu_{j}(1 \leqq j \leqq N+1)$ on $C^{n}$ with no common irreducible components, if we take entire functions $k_{j}$ whose zeros define divisors $\nu_{j}$, the set of meromorphic maps $f$ with $\nu\left(f, H_{j}\right)=\nu_{j}(1 \leqq j \leqq N+1)$ consists of maps

$$
f=e^{g_{1}} k_{1}: e^{g_{2}} k_{2}: \cdots: e^{g_{N+1}} k_{N+1},
$$

where $g_{1}, \cdots, g_{N+1}$ are arbitrary entire functions.

We shall give in $\S 2$ the precise formulation of the above-mentioned main result and its proof. In the process of the proof, we need a basic lemma (Lemma 2.9). It will be proved in $\S 3$.

\section{§2. Main Theorem}

We recall first some notations and terminology in the previous papers. For a non-zero holomorphic function $f$ on a domain $D$ in $C^{n}$, an integervalued function $\nu_{f}$ on $D$ is defined as

$$
\nu_{f}(a)=\min \left\{m ; P_{m}(u) \not \equiv 0\right\}
$$

for each point $a=\left(a_{1}, \cdots, a_{n}\right)$ in $D$ if $f$ is expanded as convergent series

$$
f\left(u_{1}+a_{1}, \cdots, u_{n}+a_{n}\right)=\sum_{m \geqq 0} P_{m}\left(u_{1}, \cdots, u_{n}\right)
$$

around $a$, where $P_{m}(u)$ is identically zero or a homogeneous polynomial of degree $m$. An integer-valued function $\nu$ on a domain $D$ in $C^{n}$ is called to be a divisor on $D$ if it can be written

$$
\nu=\nu_{g}-\nu_{h}
$$


with non-zero holomorphic functions $g$ and $h$ on a neighborhood of each point in $D$. For a divisor $\nu$ on $D$ the set

$$
|\nu|:=\{\overline{z \in D ; \nu(z) \neq 0\}} \cap D
$$

is an analytic set in $D$, which we call the support of $\nu$.

Let $f$ be a meromorphic map of $C^{n}$ into $P^{N}(C)$. Taking homogeneous. coordinates $w_{1}: w_{2}: \cdots: w_{N+1}$ on $P^{N}(C)$ arbitrarily, we can write

$$
f=f_{1}: f_{2}: \cdots: f_{N+1}
$$

with holomorphic functions $f_{1}, \cdots, f_{N+1}$ on $C^{n}$ such that

$$
\operatorname{codim}\left\{z \in C^{n} ; f_{1}(z)=\cdots=f_{N+1}(z)=0\right\} \geqq 2 .
$$

In the followings, such a representation will be called a reduced representation of $f$. Take a hyperplane

$$
H: a^{1} w_{1}+a^{2} w_{2}+\cdots+a^{N+1} w_{N+1}=0
$$

in $P^{N}(C)$ with $f\left(C^{n}\right) \not \subset H$. With the use of a holomorphic function

$$
F:=a^{1} f_{1}+a^{2} f_{2}+\cdots+a^{N+1} f_{N+1},
$$

we define a divisor,

$$
\nu(f, H):=\nu_{F}
$$

on $C^{n}$, which is determined independently of any choice of homogeneous. coordinates and reduced representations.

To state Main Theorem, we give another definition.

Definition 2.1. Meromorphic maps $f^{1}, \cdots, f^{k}$ of $C^{n}$ into $P^{N}(C)$ are called to be algebraically independent if the image of a meromorphic map.

$$
f^{1} \times f^{2} \times \cdots \times f^{k}: C^{n} \rightarrow P^{N}(C)^{k}:=P^{N}(C) \times \cdots \times P^{N}(C) \quad(k \text { times })
$$

is not included in any proper algebraic subset of $P^{N}(C)^{k}$, where $f^{1} \times \cdots$ $\times f^{k}$ is a meromorphic map defined by $\left(f^{1} \times \cdots \times f^{k}\right)(z)=\left(f^{1}(z), \cdots, f^{k}(z)\right)$ for generic points $z$ in $\boldsymbol{C}^{n}$.

Now, we give

MaIn Theorem. Let $H_{1}, \cdots, H_{N+2}$ be hyperplanes in general position. in $P^{N}(C)$ and $\nu_{1}, \cdots, \nu_{N+2}$ be divisors whose supports have no common irreducible components. Consider the set $\mathscr{F}$ of all meromorphic maps $f$ of 
$C^{n}$ into $P^{N}(C)$ such that $f\left(C^{n}\right) \not \subset H_{j}$ and $\nu_{j}=\nu\left(f, H_{j}\right)$ for $j=1,2, \cdots, N+2$. If $\mathscr{F}$ contains $N+1$ algebraically independent maps $f^{1}, \cdots, f^{N+1}$, then $\mathscr{F}$ contains no element other than $f^{1}, \cdots, f^{N+1}$.

Remark. In the case $N=1, \# \mathscr{F} \leqq 2$ by the H. Cartan's theorem stated in $\S 1$, where $\# \mathscr{F}$ denotes the number of elements of $\mathscr{F}$. The author does not know even in the case $N=2$ whether $\# \mathscr{F}<\infty$ or not.

For the proof of Main Theorem, we suppose that $\mathscr{F}$ contains algebraically independent maps $f^{1}, \cdots, f^{N+1}$. Our aim is to show that any element in $\mathscr{F}$, say $f^{N+2}$, is necessarily equal to one of $f^{1}, f^{2}, \cdots, f^{N+1}$.

Choose homogeneous coordinates $w_{1}: w_{2}: \cdots: w_{N+1}$ on $P^{N}(C)$ such that

$$
\begin{array}{ll}
H_{j}: w_{\jmath}=0 & (1 \leqq j \leqq N+1) \\
H_{N+2}: w_{1}+w_{2}+\cdots+w_{N+1}=0 &
\end{array}
$$

for given hyperplanes $H_{j}(1 \leqq j \leqq N+2)$ in general position and take reduced representations

$$
f^{i}=f_{1}^{i}: f_{2}^{i}: \cdots: f_{N+1}^{i}
$$

for $i=1,2, \cdots, N+2$. For each $\nu_{j}(1 \leqq j \leqq N+2)$ we can find a nonzero holomorphic function $k_{j}$ on $C^{n}$ such that $\nu_{k_{j}}=\nu_{j}$. By the assumption $\nu\left(f, H_{j}\right)=\nu_{j}$, there are non-zero holomorphic functions $h_{j}^{i}(1 \leqq i, j \leqq N+2)$ on $C^{n}$ such that

$$
\begin{array}{ll}
f_{j}^{i}=h_{j}^{i} k_{j} & (1 \leqq i \leqq N+2,1 \leqq j \leqq N+1) \\
f_{1}^{i}+\cdots+f_{N+1}^{i}=h_{N+2}^{i} k_{N+1} & (1 \leqq i \leqq N+2)
\end{array}
$$

We may choose here, instead of (2.3), other reduced representations

$$
f^{i}=h^{i} f_{1}^{i}: h^{i} f_{2}^{i}: \cdots: h^{i} f_{N+1}^{i} \quad(1 \leqq i \leqq N+2)
$$

for arbitrary nowhere zero holomorphic functions $h^{i}$ on $\boldsymbol{C}^{n}$. This means that $h_{j}^{i}$ may be replaced by $h_{j}^{i} / h^{i}$. On the other hand, we may use, instead of the above $k_{j}, h_{j} k_{j}$ for arbitrary nowhere zero holomorphic functions $h_{j}$ on $C^{n}$. Therefore, $h_{j}^{i}$ may be changed with $h_{j}^{i} / h_{j}$.

The identities (2.4) can be rewritten as

$$
h_{1}^{i} k_{1}+h_{2}^{i} k_{2}+\cdots+h_{N+1}^{i} k_{N+1}=h_{N+2}^{i} k_{N+2} \quad(1 \leqq i \leqq N+2) .
$$

By eliminating $k_{1}, \cdots, k_{N+2}$ from these identities, we obtain

$$
\operatorname{det}\left(h_{j}^{i} ; 1 \leqq i, j \leqq N+2\right) \equiv 0
$$


As in the previous papers, we consider the multiplicative group $\boldsymbol{H}^{*}$ of all nowhere zero holomorphic functions on $\boldsymbol{C}^{n}$ and the factor group $G:=H^{*} / C^{*}$, where $C^{*}:=C-\{0\}$ is the subgroup of $H^{*}$ consisting of all non-zero constant functions. We denote by $[h]$ the class in $G$ containing an element $h \in \boldsymbol{H}^{*}$ and $h \sim h^{\prime}$ if $[h]=\left[h^{\prime}\right]$ for $h, h^{\prime} \in \boldsymbol{H}^{*}$.

For our purpose, we need the following E. Borel's theorem and its consequence.

ThEOREM 2.7 ([1]). If $h_{1}, \cdots, h_{p} \in H^{*}$ satisfy an identity

$$
h_{1}+h_{2}+\cdots+h_{p} \equiv 0 \text {, }
$$

then, for any $h_{i}$, there exists some $h_{j}(i \neq j)$ with $h_{i} \sim h_{j}$, namely, $h_{i} / h_{j}$ 三const.

Theorem 2.8 ([3], Proposition 4.5). Let $h_{1}, \cdots, h_{t}$ be in $H^{*}$. If $\left[h_{1}\right]$, $\cdots,\left[h_{t}\right]$ are linearly independent over $Z$, then $h_{1}, \cdots, h_{t}$ are algebraically independent, i.e., there is no non-zero polynomial $P\left(u_{1}, \cdots, u_{t}\right)$ with $P\left(h_{1}\right.$, $\left.\cdots, h_{t}\right) \equiv 0$.

Now, let us consider arbitrary two square matrices $\mathscr{H}=\left(h_{j}^{i}\right)$ and $\mathscr{K}$ $=\left(k_{j}^{i}\right)$ of degree $N+2$ with components in $\boldsymbol{H}^{*}$. We shall call $\mathscr{H}$ and $\mathscr{K}$ to be equivalent if $\mathscr{K}$ can be obtained by the following transformations;

(i) multiplying a row or a column of $\mathscr{H}$ by a common element in $\boldsymbol{H}^{*}$,

(ii) interchanging two rows or two columns,

(iii) repeating these transformations (i), (ii) finitely many times.

For a given $\mathscr{H}=\left(h_{j}^{i}\right)\left(h_{j}^{i} \in \boldsymbol{H}^{*}\right)$, we shall call $\mathscr{K}=\left(k_{j}^{i}\right)\left(k_{j}^{i} \in \boldsymbol{H}^{*}\right)$ an admissible representation of $\mathscr{H}$ if $\mathscr{H}$ is equivalent to $\mathscr{K}$ and $k_{N+2}^{i} \equiv k_{j}^{N+2} \equiv 1$ $(1 \leqq i, j \leqq N+2)$. Evidently, any matrix $\left(h_{j}^{i}\right)\left(h_{j}^{i} \in \boldsymbol{H}^{*}\right)$ has an admissible representation. Though admissible representations of $\mathscr{H}=\left(h_{j}^{i}\right)$ are not unique,

$$
\left\{\left\{\left[k_{j}^{i}\right] ; 1 \leqq i, j \leqq N+2\right\}\right\}_{Z}=\left\{\left\{\left[\tilde{k}_{j}^{i}\right] ; 1 \leqq i, j \leqq N+2\right\}\right\}_{Z}
$$

if $\mathscr{K}=\left(k_{j}^{i}\right)$ and $\tilde{\mathcal{K}}=\left(\tilde{k}_{j}^{i}\right)$ are both admissible representations of $\mathscr{H}$, where $\left\{\left\{\left[k_{j}^{i}\right]\right\}\right\}_{Z}$ denotes the subgroup of $G$ generated by $\left[k_{j}^{i}\right]$ over $Z$. For a matrix $\mathscr{H}=\left(h_{j}^{i}\right)\left(h_{j}^{i} \in \boldsymbol{H}^{*}\right)$, taking an admissible representation $\mathscr{K}=\left(k_{j}^{i}\right)$ of $\mathscr{H}$, we define

$$
t(\mathscr{H}):=t\left(h_{j}^{i}\right):=\operatorname{rank}\left\{\left\{\left[k_{j}^{i}\right]\right\}\right\}_{z} .
$$


We give here a lemma which is basic in the proof of Main Theorem.

LEMMA 2.9. Let $\mathscr{H}=\left(h_{j}^{i} ; 1 \leqq i, j \leqq N+2\right)$ be a matrix with components in $H^{*}$ and assume that $\operatorname{det} \mathscr{H}=0$. Then,

(i) $t(\mathscr{H}) \leqq N^{2}+N$,

(ii) $t(\mathscr{H})=N^{2}+N$ only when two rows or two columns of $\mathscr{H}$ are equal.

For a while, we accept Lemma 2.9 without proof, which will be proved in the next section.

We continue the proof of Main Theorem. Let us study the matrix $\mathscr{H}=\left(h_{j}^{i} ; 1 \leqq i, j \leqq N+2\right)$ consisting of the functions $h_{j}^{i}$ satisfying the condition (2.4). Here, it may be assumed that $h_{N+2}^{i} \equiv h_{j}^{N+2} \equiv 1$ for $i, j=1$, $2, \cdots, N+2$ by suitable choices of indices $i, j$, reduced representations of $f^{i}$ and functions $k_{j}$ with $\nu_{k_{j}}=\nu_{j}$. And, it holds that

$$
\operatorname{det}\left(h_{1}^{i}, \cdots, h_{j-1}^{i}, h_{j+1}^{i}, \cdots, h_{N+2}^{i} ; 1 \leqq i \leqq N+1\right) \not \equiv 0
$$

for each $j=1,2, \cdots, N+2$. In fact, if not,

$$
\begin{aligned}
& \operatorname{det}\left(f_{1}^{i}, \cdots, f_{j-1}^{i}, f_{j+1}^{i}, \cdots, f_{N+1}^{i}, f_{1}^{i}+\cdots+f_{N+1}^{i} ; 1 \leqq i \leqq N+1\right) \\
&=(-1)^{N-j} \operatorname{det}\left(f_{1}^{i}, \cdots, f_{N+1}^{i} ; 1 \leqq i \leqq N+1\right) \\
& \equiv 0
\end{aligned}
$$

which contradicts the assumption that $f^{1}, \cdots, f^{N+1}$ are algebraically independent.

Take functions $\eta_{1}, \cdots, \eta_{t}$ in $H^{*}$ such that $\left[\eta_{1}\right], \cdots,\left[\eta_{t}\right]$ give a basis of $\left\{\left\{\left[h_{j}^{i}\right] ; 1 \leqq i, j \leqq N+2\right\}\right\}_{Z}$, where $t=t(\mathscr{H})$. Each $h_{j}^{i}$ has a representation

$$
h_{j}^{i}=c_{i j} \eta_{1}^{\ell_{i j}^{1}} \cdots \eta_{t}^{\ell_{i j}^{t}},
$$

where $c_{i j} \in C^{*}$ and $\ell_{i j}^{s} \in Z$. Consider rational functions

$$
\varphi_{j}^{i}(u)=c_{i j} u_{1}^{\ell_{i j}^{1}} \cdots u_{t}^{\ell_{i j}^{t}}
$$

of variables $u=\left(u_{1}, \cdots, u_{t}\right)$ and homogeneous linear equations

$$
\varphi_{1}^{i}(u) w_{1}+\cdots+\varphi_{N+1}^{i}(u) w_{N+1}=\varphi_{N+2}^{i}(u) w_{N+2} \quad(1 \leqq i \leqq N+2)
$$

with coefficients in the field of rational functions of $u_{1}, \cdots, u_{t}$ and $N+2$ unknowns $w_{1}, \cdots, w_{N+2}$. We have here by (2.10)

$$
\Phi_{j}(u):=(-1)^{j-1} \operatorname{det}\left(\varphi_{1}^{i}, \cdots, \varphi_{j-1}^{i}, \varphi_{j+1}^{i}, \cdots, \varphi_{N+2}^{j} ; 1 \leqq i \leqq N+2\right) \not \equiv 0
$$


because $\eta_{1}, \cdots, \eta_{t}$ are algebraically independent by virtue of Theorem 2.8. A solution of the equation (2.12) is given by

$$
\begin{aligned}
& w_{j}=\Phi_{j}\left(u_{1}, \cdots, u_{t}\right) \quad(1 \leqq j \leqq N+1) \\
& w_{N+2}=-\Phi_{N+2}\left(u_{1}, \cdots, u_{t}\right)
\end{aligned}
$$

and any other solution is obtained from them by common multiplications of a non-zero rational function. Put

$$
\Psi_{j}^{i}\left(u_{1}, \cdots, u_{t}\right):=\varphi_{j}^{i}\left(u_{1} \cdots, u_{t}\right) \Phi_{j}\left(u_{1}, \cdots, u_{t}\right)
$$

for $i=1,2, \cdots, N+2$ and $j=1,2, \cdots, N+1$. For each $i$, multiplying $\Psi_{1}^{i}, \cdots, \Psi_{N+1}^{i}$ by a common function and introducing a new variable $u_{t+1}$, we can construct homogeneous polynomials $\tilde{\Psi}_{1}^{i}\left(u_{1}, \cdots, u_{t+1}\right), \cdots, \tilde{\Psi}_{N+1}^{i}\left(u_{1}\right.$, $\cdots, u_{t+1}$ ) of the same degree such that

$$
\begin{aligned}
& \Psi_{1}^{i}\left(u_{1}, \cdots, u_{t}\right): \cdots: \Psi_{N+1}^{i}\left(u_{1}, \cdots, u_{t}\right) \\
& \quad=\tilde{\Psi}_{1}^{i}\left(u_{1}, \cdots, u_{t}, 1\right): \cdots: \tilde{\Psi}_{1}^{i}\left(u_{1}, \cdots, u_{t}, 1\right) .
\end{aligned}
$$

Let us consider a rational map $\Psi: P^{t}(C) \rightarrow P^{N}(C)^{N+2}$ such that

$$
\Psi(\tilde{u})=\left(\tilde{\Psi}_{1}^{1}(\tilde{u}): \cdots: \tilde{\Psi}_{N+1}^{1}(\tilde{u}), \cdots, \tilde{\Psi}_{1}^{N+2}(\tilde{u}): \cdots: \tilde{\Psi}_{N+1}^{N+2}(\tilde{u})\right)
$$

for each $\tilde{u}=u_{1}: \cdots: u_{t}: u_{t+1}$ in $P^{t}(C)$ except a nowhere dense algebraic set

$$
\bigcup_{j=1}^{N+2}\left\{\tilde{u} \in P^{t}(C) ; \tilde{\Psi}_{1}^{i}(\tilde{u})=\cdots=\tilde{\Psi}_{N+1}^{i}(\tilde{u})=0\right\} .
$$

The image $W=\Psi\left(P^{t}(C)\right)$ is an algebraic subvariety of $P^{N}(C)^{N+2}$ with $\operatorname{dim} W \leqq t . \quad$ Substitute $u_{1}=\eta_{1}, \cdots, u_{t}=\eta_{t}$ in (2.12). The equations (2.12) become

$$
h_{1}^{i} w_{1}+h_{2}^{i} w_{2}+\cdots+h_{N+1}^{i} w_{N+1}=h_{N+2}^{i} w_{N+2} \quad(1 \leqq i \leqq N+2)
$$

and $w_{1}=k_{1}, \cdots, w_{N+2}=k_{N+2}$ satisfy these equations because of (2.5). We have therefore

$$
k_{1}: k_{2}: \cdots: k_{N+2}=\Phi_{1}\left(\eta_{1}, \cdots, \eta_{t}\right): \cdots:-\Phi_{N+2}\left(\eta_{1}, \cdots, \eta_{t}\right) .
$$

And, by (2.13),

$$
\begin{aligned}
& \tilde{\Psi}_{1}^{i}\left(\eta_{1}, \cdots, \eta_{t}, 1\right): \cdots: \tilde{\Psi}_{N+1}^{i}\left(\eta_{1}, \cdots, \eta_{t}, 1\right) \\
&=\Psi_{1}^{i}\left(\eta_{1}, \cdots, \eta_{t}\right): \cdots: \Psi_{N+1}^{i}\left(\eta_{1}, \cdots, \eta_{t}\right) \\
&=h_{1}^{i} k_{1}: \cdots: h_{N+1}^{i} k_{N+1} \\
&=f_{1}^{i}: \cdots: f_{N+1}^{i}
\end{aligned}
$$


for each $i=1,2, \cdots, N+2$. We can conclude from (2.14)

$$
\Psi\left(\eta_{1}: \cdots: \eta_{t}: 1\right)=\left(f_{1}^{1}: \cdots: f_{N+1}^{1}, \cdots, f_{1}^{N+2},: \cdots: f_{N+1}^{N+2}\right) .
$$

This means that

$$
\Psi \circ \eta=f^{1} \times \cdots \times f^{N+2}
$$

for a map $\eta:=\eta_{1}: \eta_{2}: \cdots: \eta_{t}: 1$ of $C^{n}$ into $P^{t}(C)$. Therefore, the image of the map $f^{1} \times \cdots \times f^{N+1}: C^{n} \rightarrow P^{N}(C)^{N+2}$ is included in $W$.

Let $\pi: P^{N}(C)^{N+2} \rightarrow P^{N}(C)^{N+1}$ be the projection onto the first $N+1$ components. The image $\pi(W)$ of $W$ is an algebraic subset of $P^{N}(C)^{N+1}$ and

$$
\operatorname{dim} \pi(W) \leqq \operatorname{dim} W \leqq t
$$

On the other hand, the image of $f^{1} \times \cdots \times f^{N+1}: C^{n} \rightarrow P^{N}(C)^{N+1}$ is obviously included in $\pi(W)$ and it cannot be included in any proper subvariety of $P^{N}(C)^{N+1}$. Therefore, $\operatorname{dim} \pi(W)=N^{2}+N \leqq t$. We know $t \leqq N^{2}+N$ by Lemma 2.9, (i). In conclusion, $t=N^{2}+N$. In this case, by Lemma 2.9, (ii), two rows or two columns of $\mathscr{H}=\left(h_{j}^{i}\right)$ are equal. If two rows are equal, e.g., $h_{j}^{i_{1}}=h_{j}^{i_{2}}(1 \leqq j \leqq N+2)$ for some distinct $i_{1}, i_{2}$, then $f^{i_{1}}=f^{i_{2}}$. Since any two of $f^{1}, \cdots, f^{N+1}$ are not equal by the assumption, we can conclude that $f^{N+2}$ is equal to some $f^{i}(1 \leqq i \leqq N+1)$. And, if two columns are equal, then $f_{j_{1}}^{i}=f_{j_{2}}^{i}(1 \leqq i \leqq N+2)$ for some $j_{1}, j_{2}$ with $1 \leqq j_{1}$ $<j_{2} \leqq N+1$ or $f_{j}^{i}=f_{1}^{i}+\cdots+f_{N+1}^{i}(1 \leqq i \leqq N+2)$ for some $j$ with $1 \leqq j$ $\leqq N+1$. In any case, $f^{i}$ themselves are algebraically degenerate. This contradicts the assumption. We have thus Main Theorem.

\section{§3. Proof of a basic lemma}

We proceed to the proof of Lemma 2.9 stated in the previous section. Let $\mathscr{H}=\left(h_{j}^{i}: 1 \leqq i, j \leqq N+2\right)\left(h_{j}^{i} \in H^{*}\right)$ and satisfy the condition

$$
\operatorname{det}\left(h_{j}^{i} ; 1 \leqq i, j \leqq N+2\right) \equiv 0 .
$$

The proof is given by the induction on $N$. For the case $N=1$, we have the more precise result.

LEMmA 3.2. Let $\mathscr{H}=\left(h_{j}^{i} ; 1 \leqq i, j \leqq 3\right)\left(h_{j}^{i} \in H^{*}\right)$ satisfy the condition $\operatorname{det} \mathscr{H}=0$. Then,

(i) two rows or two columns of $\mathscr{H}$ are mutually proportional or

(ii) $h_{j}^{i} / h_{\ell}^{k} \equiv$ const. for any $i, j, k, \ell$. 
This was given by H. Cartan ([2]). He proved the theorem stated in $\S 1$ by the aid of this fact. To make our statements self-contained, we describe here its proof and, in addition, the proof of the CartanNevanlinna's theorem.

Proof of Lemma 3.2. We study first the case that $h_{k}^{i} h_{\ell}^{j} \sim h_{\ell}^{i} h_{k}^{j}$, namely, $h_{k}^{i} h_{\ell}^{j} / h_{\ell}^{i} h_{k}^{j} \equiv$ const. for some $i, j, k, \ell$ with $i \neq j, k \neq \ell$. It suffices to prove Lemma 3.2 for an admissible representation of $\mathscr{H}$. So, we may put

$$
\mathscr{H}=\left(\begin{array}{ccc}
h_{1} & h_{2} & 1 \\
h_{3} & h_{4} & 1 \\
1 & 1 & 1
\end{array}\right) \quad\left(h_{i} \in \boldsymbol{H}^{*}\right)
$$

and assume $c:=h_{4} \in C^{*}$. By the assumption,

$$
(c-1) h_{1}+h_{2}+h_{3}-h_{2} h_{3}-c=0 .
$$

Suppose that $h_{2} \nsim 1$ and $h_{3} \nsim 1$. Then, by Theorem 2.7 , we have necessarily $h_{2} \sim h_{3}$, since $h_{2} \sim h_{1}$ and $h_{3} \sim h_{1}$ even if we assume $h_{2} \not h_{3}$ because $h_{2}$ $\not h_{2} h_{3}, h_{3} \not h_{2} h_{3}$. Moreover, $h_{2} h_{3} \sim 1$ and so $h_{2}^{2} \sim h_{2} h_{3} \sim 1$. This is a contradiction. We have therefore $h_{2} \sim 1$ or $h_{3} \sim 1$. We examine the case $h_{2} \sim 1$ only because the proof of the other case is similar. Put $d:=h_{2}$. Applying Theorem 2.7 to the identity

$$
(c-1) h_{1}-(d-1) h_{3}+d-c=0,
$$

we see easily $h_{1} \sim h_{3} \sim 1$ or $c=d=1$. In any case, we have Lemma 3.2.

Let us study next the case $h_{k}^{i} h_{\ell}^{j} \not h_{\ell}^{i} h_{k}^{j}$ for any $i, j, k, \ell$ with $i \neq j, k \neq \ell$. By the help of Theorem 2.7, for the first term $h_{1}^{1} h_{2}^{2} h_{3}^{3}$ in the identity

$$
h_{1}^{1} h_{2}^{2} h_{3}^{3}+h_{2}^{1} h_{3}^{2} h_{1}^{3}+h_{3}^{1} h_{1}^{2} h_{2}^{3}-h_{1}^{1} h_{3}^{2} h_{2}^{3}-h_{3}^{1} h_{2}^{2} h_{1}^{3}-h_{2}^{1} h_{1}^{2} h_{3}^{3} \equiv 0,
$$

there is some other term $h_{j_{1}}^{1} h_{j_{2}}^{2} h_{j_{3}}^{3}$ with $h_{1}^{1} h_{2}^{2} h_{3}^{3} \sim h_{j_{1}}^{1} h_{j_{2}}^{2} h_{j_{3}}^{3}$, which cannot be equal to the last three terms by the assumption. The same argument remains valid for the terms $h_{2}^{1} h_{3}^{2} h_{1}^{3}$ and $h_{3}^{1} h_{1}^{2} h_{2}^{3}$. In conclusion,

$$
h_{1}^{1} h_{2}^{2} h_{3}^{3} \sim h_{2}^{1} h_{3}^{2} h_{1}^{3} \sim h_{3}^{1} h_{1}^{2} h_{2}^{3}
$$

and

$$
h_{1}^{1} h_{3}^{2} h_{2}^{3} \sim h_{3}^{1} h_{2}^{2} h_{1}^{3} \sim h_{2}^{1} h_{1}^{2} h_{3}^{3}
$$

We may assume $\mathscr{H}$ is written as (3.3). Then

$$
h_{1} h_{4} \sim h_{2} \sim h_{3}, \quad h_{2} h_{3} \sim h_{1} \sim h_{4} .
$$


This implies

$$
h_{1} \sim\left(h_{1} h_{4}\right)^{2} \sim h_{1}^{4}
$$

and so $h_{1} \sim h_{2} \sim h_{3} \sim h_{4} \sim 1$. We get Lemma 3.2 in this case too.

Proof of the Cartan-Nevanlinna's theorem. Let $\varphi, \psi, \chi$ be non-constant meromorphic functions on $C$ and suppose that $\varphi(z)-a_{i}, \psi(z)-a_{i}, \chi(z)-a_{t}$ have the same zeros with the same multiplicities for three distinct values $a_{j}(i=1,2,3)$. Our aim is to show $\varphi=\psi, \psi=\chi$ or $\chi=\varphi$. We may regard $\varphi, \psi, \chi$ as holomorphic maps of $C$ into $P^{1}(C)$ and write $a_{1}=0: 1, a_{2}$ $=1: 0$ and $a_{3}=1:-1$ with homogeneous coordinates on $P^{1}(C)$. Take reduced representations

$$
\varphi=\varphi_{1}: \varphi_{2}, \quad \psi=\psi_{1}: \psi_{2}, \quad \chi=\chi_{1}: \chi_{2}
$$

and define

$$
\begin{aligned}
& h_{1}^{1}=h_{2}^{1}=h_{3}^{1}=1, \\
& h_{1}^{2}=\frac{\psi_{1}}{\varphi_{1}}, \quad h_{2}^{2}=\frac{\psi_{2}}{\varphi_{2}}, \quad h_{3}^{2}=\frac{\psi_{1}+\psi_{2}}{\varphi_{1}+\varphi_{2}}, \\
& h_{1}^{3}=\frac{\chi_{1}}{\varphi_{1}}, \quad h_{2}^{3}=\frac{\chi_{2}}{\varphi_{2}} \cdot h_{3}^{3}=\frac{\chi_{1}+\chi_{2}}{\varphi_{1}+\varphi_{2}} .
\end{aligned}
$$

By the assumption, they are nowhere zero holomorphic functions on $C$ and $\operatorname{det}\left(h_{j}^{i} ; 1 \leqq i, j \leqq 3\right) \equiv 0$. So, we have the conclusion (i) or (ii) of Lemma 3.2. In any case, we obtain easily the desired conclusion.

We go back to the proof of Lemma 2.9. Suppose that Lemma 2.9 is true for matrices of degree $\leqq N+1$ and consider a matrix $\mathscr{H}=\left(h_{j}^{i}\right)$ of degree $N+2$ satisfying the condition (3.1), where $N \geqq 2$. The group $G$ $=H^{*} / C^{*}$ is a torsionfree abelian group. For any $\eta \in H^{*}$ and $\ell \in Z-\{0\}$ we can find some $\eta^{\prime} \in H^{*}$ such that $\left(\eta^{\prime}\right)^{\ell}=\eta$. So, $G$ is considered as a vector space over $\boldsymbol{Q}$. Suitable $t$ elements $h_{j_{1}}^{i_{1}}, h_{j_{2}}^{i_{2}}, \cdots, h_{j_{t}}^{i_{t}}$ in $\left\{h_{j}^{i} ; 1 \leqq i\right.$, $j \leqq N+2\}$ give a basis of $\left\{\left\{\left[h_{j}^{i}\right]\right\}\right\}_{Q}$, where $t=t\left(h_{j}^{i}\right)=\operatorname{dim}_{\boldsymbol{Q}}\left\{\left\{\left[h_{j}^{i}\right]\right\}\right\}_{\boldsymbol{Q}}$. Then, there are $t$ functions $\eta_{1}, \cdots, \eta_{t}$ in $H^{*}$ such that $\left[\eta_{1}\right], \cdots,\left[\eta_{t}\right]$ are linearly independent over $Z$ and functions $h_{j}^{i}$ can be written as

$$
h_{j_{s}}^{i_{s}}=\eta_{s}^{\ell_{s}}
$$

for $s=1,2, \cdots, t$ and

$$
h_{j}^{i}=c_{i j} \eta_{1}^{\ell_{i j}^{1}} \cdots \eta_{t}^{\ell_{t}^{t}}
$$


for the other $h_{j}^{i}$ 's, where $\ell_{s}$ are positive integers and $c_{i j} \in C^{*}, \ell_{i j}^{s} \in Z$. Here, the choice of $\left\{i_{1}, j_{1}, \cdots, i_{t}, j_{t}\right\}$ is not unique. We choose some and fix them for a while. For the sake of convenience, we put $I:=\{(i, j): 1 \leqq j$, $j \leqq N+1\}, I_{1}:=\left\{\left(i_{1}, j_{1}\right), \cdots,\left(i_{t}, j_{t}\right)\right\}(\subset I)$ and $I_{2}:=I-I_{1}$.

Consider rational functions of $t$ independent variables $u=\left(u_{1}, \cdots, u_{t}\right)$

$$
\begin{aligned}
& \varphi_{j_{s}}^{i_{s}}(u)=u_{s}^{\ell_{s}} \quad(1 \leqq s \leqq t) \\
& \varphi_{j}^{i}(u)=c_{i j} u_{1}^{\ell_{i j}^{1}} \cdots u_{t}^{\ell_{i j}^{t}} \quad\left((i, j) \in I_{2}\right)
\end{aligned}
$$

and $\varphi_{N+2}^{i}(u)=\varphi_{j}^{N+2}(u)=1(1 \leqq i, j \leqq N+2)$. Substitute (3.4) and (3.5) into the identity (3.1). Then, we have an identity

$$
\operatorname{det}\left(\varphi_{j}^{i}(u) ; 1 \leqq i, j \leqq N+2\right) \equiv 0,
$$

because $\eta_{1}, \cdots, \eta_{t}$ are algebraically independent.

Now, to prove Lemma 2.9 , (i), assume that $t \geqq N^{2}+N+1$. Then,

$$
\# I_{2}=(N+1)^{2}-t \leqq(N+1)^{2}-\left(N^{2}+N+1\right)=N .
$$

Accordingly, it is easily seen that there exists some $j_{0}$ with $1 \leqq j_{0} \leqq N+1$ such that $\left(i, j_{0}\right) \in I_{1}$ for any $i=1,2, \cdots, N+1$. Changing indices, we may assume $j_{0}=N+1$ and $\left(i_{1}, j_{1}\right)=(1, N+1), \cdots,\left(i_{N+1}, j_{N+1}\right)=(N+1$, $N+1)$. Additionally, if $I_{2} \neq \phi$, we choose indices such that $(N+1, N) \in I_{2}$.

$$
N_{0}:=\sharp\left\{(i, j) \in I_{1} ; 1 \leqq i, j \leqq N\right\} \geqq N^{2}-N+1 .
$$

Indeed, $N_{0}=(N+1)^{2}>N^{2}-N+1$ in the case $I_{2}=\phi$ and $N_{0} \geqq t-2 N \geqq$ $N^{2}-N+1$ in the case $I_{2} \neq \phi$. Substitute $u_{1}=\cdots=u_{N}=1$ and $u_{s}=\eta_{s}$ for $s=N+1, \cdots, t$ into (3.6). We have then

$$
\operatorname{det}\left(\tilde{h}_{j}^{i} ; i, j=1,2, \cdots, N, N+2\right) \equiv 0,
$$

where $\tilde{h}_{j}^{i}:=\varphi_{j}^{i}\left(1, \cdots, 1, \eta_{N+1}, \cdots, \eta_{t}\right) \in \boldsymbol{H}^{*}$. By the induction hypothesis,

$$
t\left(\tilde{h}_{j}^{i} ; i, j=1,2, \cdots, N, N+2\right) \leqq N^{2}-N .
$$

However, we see easily $N_{0} \leqq t\left(\tilde{h}_{j}^{i} ; i, j=1, \cdots, N, N+2\right)$. This contradicts (3.7). We have thus Lemma 2.9, (i).

For the proof of Lemma 2.9, (ii), assume next $t=N^{2}+N$. We study first

The case A. We can choose the above $I_{1}=\left\{\left(i_{1}, j_{1}\right), \cdots,\left(i_{t}, j_{t}\right)\right\}$ such that there is at least one row or one column whose indices are all in $I_{1}$.

By exchanging rows and columns and changing indices if necessary, it may be assumed that 


$$
\{(1, N+1),(2, N+1), \cdots,(N+1, N+1)\} \subseteq I_{1} .
$$

Then, there is another column such that at most one of its indices is in $I_{2}$. For, if not, $N+1=\# I_{2} \geqq 2 N>N+1$ because $N \geqq 2$. Without loss of generality, it may be assumed that

$$
\{(1, N),(2, N), \cdots,(N, N)\} \subseteq I_{1}
$$

and $\left(N+1, j_{0}\right) \in I_{2}$ for some $j_{0}$ with $1 \leqq j_{0} \leqq N$. Moreover, we choose indices of $u_{s}$ such that $\varphi_{N+1}^{i}(u)=u_{i}^{\ell_{i}}$ for $i=1,2, \cdots, N+1$ as before. Putting $u_{1}=\cdots=u_{N}=1$ and $u_{s}=\eta_{s}(N+1 \leqq s \leqq t)$ in (3.6), we get again (3.8). Since $\sharp\left\{(i, j) \in I_{1} ; j=N+1\right\}=N+1$ and $\sharp\left\{(i, j) \in I_{1} ; i=N\right.$ $+1,1 \leqq j \leqq N\} \leqq N-1, t(\widetilde{\mathscr{H}}) \geqq t-((N+1)+(N-1))=N^{2}-N$, where $\widetilde{\mathscr{H}}=\left(\tilde{h}_{j}^{i} ; i, j=1,2, \cdots, N, N+2\right)$. On the other hand, $t(\widetilde{\mathscr{H}}) \leqq N^{2}-N$ by the induction hypothesis. So, $t(\widetilde{\mathscr{H}})=N^{2}-N$ and, hence, two rows or two columns in $\widetilde{\mathscr{H}}$ are equal. Since $\tilde{h}_{N}^{i_{1}} \neq \tilde{h}_{N}^{i_{2}}$ for any $i_{1}, i_{2}$ with $1 \leqq i_{1}<i_{2} \leqq N$, two rows in $\breve{\mathscr{H}}$ cannot be equal. Consequently, there are indices $j_{1}, j_{2}$ with $1 \leqq j_{1}<j_{2} \leqq N$ or $j_{1}=N+2,1 \leqq j_{2} \leqq N$ such that $\tilde{h}_{j_{1}}^{i}=\tilde{h}_{j_{2}}^{i}$ for $i=1,2, \cdots, N$. In the former case, as is easily seen,

$$
\sharp\left\{(i, j) \in I_{1} ; 1 \leqq i, j \leqq N\right\}=N^{2}-N
$$

and $\tilde{h}_{j}^{i}=\tilde{h}_{\ell}^{k}$ for any distinct $(i, j),(k, \ell)$ in $I_{1}$. Hence,

$$
\left\{(i, j): 1 \leqq i, j \leqq N, j \neq j_{1}, j_{2}\right\} \subseteq I_{1}
$$

and, for each $i(1 \leqq i \leqq N)$, exactly one of $\left(i, j_{1}\right)$ and $\left(i, j_{2}\right)$ is in $I_{1}$. Since $\tilde{h}_{j_{1}}^{i}=\tilde{h}_{j_{2}}^{i}$, we can write

$$
h_{j_{1}}^{i}=\eta_{s}^{\ell_{s}}, \quad h_{j_{2}}^{i}=\eta_{1}^{n_{1}} \cdots \eta_{N}^{n_{N}} \eta_{s}^{\ell_{s}}
$$

or

$$
h_{j_{1}}^{i}=\eta_{1}^{n_{1}} \cdots \eta_{N}^{n_{N}} \eta_{s}^{\ell_{s}}, \quad h_{j_{2}}^{i}=\eta_{s}^{\ell_{s}}
$$

according as $\left(i, j_{1}\right) \in I_{1}$ or $\left(i, j_{2}\right) \in I_{1}$. Changing $\eta_{s}$ by $\tilde{\eta}_{s}$ with $\tilde{\eta}_{s}^{\ell_{s}}=\eta_{1}^{n_{1}} \eta_{2}^{n_{2}} \ldots$ $\eta_{N}^{n_{N} \eta_{s}^{\ell_{s}}}$ if necessary, we may assume $\left(i, j_{1}\right) \in I_{1}$ and $\left(i, j_{2}\right) \in I_{2}$. In any case, the indices may be chosen so that $j_{2}=1$ and so

$$
\left\{(i, j) \in I_{2} ; 1 \leqq i, j \leqq N\right\}=\{(1,1),(2,1), \cdots,(N, 1)\} .
$$

For the sake of convenience, we change some notations in the followings. We denote by $u_{j}^{i}, \eta_{j}^{i}$ and $\ell_{j}^{i}$ the variable $u_{s}$, function $\eta_{s}$ and positive integer $\ell_{s}$ if $\varphi_{j}^{i}(u)=u_{s}^{\ell_{s}}$ for each $(i, j) \in I_{1}$. Substitute $u_{2}^{1}=\cdots=u_{N+1}^{1}=1$ 
and $u_{j}^{i}=\eta_{j}^{i}$ for the other $(i, j)$ in $I_{1}$ into (3.6). Subtracting the $(N+2)$-th row from the first row of $\mathscr{H}=\left(h_{j}^{i}\right)$, we obtain

$$
\left(\tilde{h}_{1}^{1}-1\right) \operatorname{det}\left(\tilde{h}_{j}^{i} ; 2 \leqq i, j \leqq N+2\right) \equiv 0,
$$

where $\tilde{h}_{j}^{i}$ are the functions obtained from $h_{j}^{i}$ by substituting $u_{2}^{1}=\cdots=u_{N+1}^{1}$ $=1$ and $u_{j}^{i}=\eta_{j}^{i}$ for the other $(i, j) \in I_{1}$. As is easily seen, for a matrix $\tilde{\mathscr{H}}:=\left(\tilde{h}_{j}^{i} ; 2 \leqq i, j \leqq N+2\right)$,

$$
t(\tilde{\mathscr{H}}) \geqq N^{2}+N-(N+1)>N^{2}-N .
$$

By the induction hypothesis, $\operatorname{det}(\tilde{\mathscr{H}}) \not \equiv 0$. Hence, we have $\tilde{h}_{1}^{1} \equiv 1$. This means that $\varphi_{1}^{1}(u)$ can be written as

$$
\varphi_{1}^{1}(u)=\left(u_{2}^{1}\right)^{m_{2}}\left(u_{3}^{1}\right)^{m_{3}} \cdots\left(u_{N+1}^{1}\right)^{m_{N+1}},
$$

where $m_{2}, m_{3}, \cdots, m_{N+1} \in Z$.

Assume that $m_{2}=\cdots=m_{N+1}=0$, namely, $\varphi_{1}^{1}(u) \equiv 1$. Put $u_{2}^{1}=\cdots$ $=u_{N}^{1}=1$ and $u_{j}^{i}=\eta_{j}^{i}$ for the other $(i, j) \in I_{1}$ in (3.6). By subtracting the $(N+2)$-th row from the first row, we have an identity

$$
\operatorname{det}\left(h_{\ell}^{k} ; \begin{array}{l}
k=2,3, \cdots, N+2 \\
\ell=1,2, \cdots, N, N+2
\end{array}\right) \equiv 0 .
$$

Let us consider next the case $\left(m_{2}, \cdots, m_{N+1}\right) \neq(0, \cdots, 0)$. Take positive integers $p_{2}, \cdots, p_{N+1}$ such that

$$
m:=m_{2} p_{2}+\cdots+m_{N+1} p_{N+1} \neq 0 .
$$

Substitute $u_{j}^{1}=v^{p_{j}}(2 \leqq j \leqq N+1)$ into (3.6), where $v$ is a new independent variable. Then, (3.6) may be regarded as an identity of rational functions of $v$ and $u_{\ell}^{k}\left(k \neq 1,(k, \ell) \in I_{1}\right)$. For the case $m>0$, put $v=0$. We have then

$$
\operatorname{det}\left(\varphi_{\ell}^{k}(u) ; \begin{array}{l}
k=2, \cdots, N+2 \\
\ell=1, \cdots, N+1
\end{array}\right) \equiv 0 .
$$

For the case $m<0$, put $v=0$ after multiplying the first row by $v^{-m}$. Then,

$$
\operatorname{det}\left(\varphi_{\ell}^{k}(u) ; k, \ell=2, \cdots, N+2\right) \equiv 0 .
$$

In any case, we obtain a minor $\operatorname{det}\left(h_{\ell}^{k}\right)$ of $\mathscr{H}=\left(h_{j}^{i} ; 1 \leqq j, j \leqq N+2\right)$ of degree $N+1$ which vanishes identically and, as is easily seen, satisfies 
the condition $t\left(h_{\ell}^{k}\right)=N^{2}-N$. By the induction hypothesis, two rows or two columns of $\left(h_{\ell}^{k}\right)$ are equal. Subtract one of them from the other in the matrix $\left(h_{j}^{i}\right)$. We can easily conclude that two rows or two columns of $\mathscr{H}$ are equal.

It remains to prove Lemma 2.9, (ii) for the following case.

The case $\mathrm{B}$. We cannot choose $I=\left\{\left(i_{1}, j_{1}\right), \cdots,\left(i_{t}, j_{t}\right)\right\}$ satisfying the condition as in the case A.

Then, for each $i_{0}$ and each $j_{0}\left(1 \leqq i_{0}, j_{0} \leqq N+1\right)$ there exist exactly one $j_{1}$ and exactly one $i_{1}$ such that $\left(i_{0}, j_{1}\right) \in I_{2}$ and $\left(i_{1}, j_{0}\right) \in I_{2}$. Changing indices suitably, we may put

$$
I_{2}=\{(1,1),(2,2), \cdots,(N+1, N+1)\} .
$$

Substitute $u_{N+1}^{1}=\cdots=u_{N+1}^{N}=1$ and $u_{j}^{i}=\eta_{j}^{i}$ for the other $(i, j) \in I_{1}$ into (3.6) and subtract the $(N+2)$-th row from the $(N+1)$-th row. We get easily

$$
\left(\tilde{h}_{N+1}^{N+1}-1\right) \operatorname{det}\left(\tilde{h}_{j}^{i} ; i, j=1,2, \cdots, N, N+2\right) \equiv 0,
$$

where $\tilde{h}_{j}^{i}$ are the functions obtained from $h_{j}^{i}$ by substituting $u_{N+1}^{1}=\cdots$ $=u_{N+1}^{N}=1$ and $u_{j}^{i}=\eta_{j}^{i}$ for the other $(i, j) \in I_{1}$. Obviously, $t(\widetilde{\mathscr{P}})=N^{2}-N$ for a matrix $\widetilde{\mathscr{H}}=\left(\tilde{h}_{j}^{i}: i, j=1,2, \cdots, N, N+2\right)$. And, any two rows or two columns of $\widetilde{\mathscr{H}}$ are not equal. For, if not, we have the case $\mathrm{A}$ by a suitable choice of $I_{1}$. By the induction hypothesis, $\operatorname{det}(\widetilde{\mathscr{H}}) \not \equiv 0$ and so $\tilde{h}_{N+1}^{N+1} \equiv 1$. This means that $\varphi_{N+1}^{N+1}(u)$ can be expressed as

$$
\varphi_{N+1}^{N+1}(u)=\left(u_{N+1}^{1}\right)^{m_{1}}\left(u_{N+1}^{2}\right)^{m_{2}} \cdots\left(u_{N+1}^{N}\right)^{m_{N}} \text {, }
$$

where $m_{1}, m_{2}, \cdots, m_{N+1} \in Z$. Put $u_{1}^{N+1}=\cdots=u_{N}^{N+1}=1, u_{j}^{i}=\eta_{j}^{i}$ for the other $(i, j) \in I_{1}$ and repeat the same argument as the above. It is shown that $\varphi_{N+1}^{N+1}(u)$ can be expressed by variables $u_{1}^{N+1}, \cdots, u_{N}^{N+1}$ only. Therefore, we get $m_{1}=\cdots=m_{N}=0$, namely, $\varphi_{N+1}^{N+1}(u) \equiv 1$. The same argument remains valid if we exchange the index $N+1$ by the other $i=1,2, \cdots, N$. So, $\varphi_{1}^{1}(u)=\cdots=\varphi_{N+1}^{N+1}(u)=1$. Now, $u_{N+1}^{2}=\cdots=u_{N+1}^{N}=1$ and $u_{j}^{i}=\eta_{j}^{i}$ for the other $(i, j) \in I_{1}$ in (3.6) and subtract the $(N+2)$-th row from the $(N+1)$-th row in (3.6). We have then

$$
\operatorname{det}\left(h_{\ell}^{k} ; \begin{array}{l}
k=2, \cdots, N+2 \\
\ell=1, \cdots, N+1
\end{array}\right) \equiv 0
$$


This contradicts the induction hypothesis, because $t\left(h_{\ell}^{k} ; \begin{array}{l}2 \leqq k \leqq N+2 \\ 1 \leqq l \leqq N+1\end{array}\right)$ $>N^{2}-N$. Therefore, the case B is impossible. This completes the proof of Lemma 2.9 .

\section{REFERENCES}

[1] E. Borel, Sur les zéros des fonctions entières, Acta Math., 20 (1897), 357-396.

[2] H. Cartan, Sur les systèmes de fonctions holomorphes a variétés linéaires lacunaires, Ann. de E.N.S., 45 (1928), 255-346.

[ 3 ] H. Fujimoto, The uniqueness problem of meromorphic maps into the complex projective space, Nagoya Math. J., 58 (1975), 1-23.

[4] - A uniqueness theorem of algebraically non-degenerate meromorphic maps into $P^{N}(C)$, Nagoya Math. J., 64 (1976), 117-147.

[5] - Remarks to the uniqueness problem of meromorphic maps into $P^{N}(C)$, I, II, Nagoya Math. J., 71 (1978), 13-41.

[6] R. Nevanlinna, Le théorèmes de Picard-Borel et la théorie des fonctions méromorphes, Gauthier-Villars, Paris, 1929.

Nagoya University 